\title{
Compressible Time-Dependent Steam Flow And Thermal Heat Analysis In Squared Cylinder: A Numerical Investigation
}

\author{
Azad Hussain ( $\nabla$ azad.hussain@uog.edu.pk) \\ University of Gujrat \\ Muhammad Arsaln \\ University of Gujrat \\ Ali Hassan \\ University of Gujrat \\ Aysha Rehman \\ University of Gujrat
}

\section{Research Article}

Keywords: Time-dependent, Compressible flow, Steam laminar flow, BDF technique.

Posted Date: December 29th, 2021

DOI: https://doi.org/10.21203/rs.3.rs-1148480/v1

License: (c) (i) This work is licensed under a Creative Commons Attribution 4.0 International License. Read Full License 


\title{
Compressible Time-dependent Steam Flow and Thermal Heat Analysis in Squared Cylinder: A Numerical Investigation
}

\author{
Azad Hussain ${ }^{1, *}$, Muhammad Arsaln ${ }^{1}$, Ali Hassan ${ }^{1, *}$, Aysha Rehman ${ }^{1}$ \\ ${ }^{1}$ University of Gujrat, Gujrat, 50700, Pakistan.
}

Corresponding author email: azad.hussain@uog.edu.pk

\begin{abstract}
This paper investigates time-dependent compressible steam laminar flow coupled with heat transfer in fluids in a squared cylinder. The present problem has been designed in COMSOLMultiphysics. The laminar flow is selected keeping the Mac number low. The flow possesses a noslip condition with the wall of geometry. The pressure kept on flow is 0 Pas and the temperature of the flow regime is 305.13 . The flow is initiated with a velocity of $0.5 \mathrm{~m} / \mathrm{s}$. The effects of time on velocity distribution and pressure distribution are described with the help of graphs. Different results like drag coefficient, lift coefficient, heat distributions are also discussed. The technique used to solve modeled problem is BDF.
\end{abstract}

Keywords: Time-dependent, Compressible flow, Steam laminar flow, BDF technique.

\section{Abbreviations:}

\begin{tabular}{clcl}
\hline $\mathrm{D}$ & Diameter of the cylinder $(\mathrm{m})$ & $U_{0}$ & Normal inflow velocity $\left(\mathrm{m}^{\mathrm{s}^{-1}}\right)$ \\
\hline $\mathrm{F}_{\mathrm{D}}$ & Drag force $\left(\mathrm{kgms}^{-2}\right)$ & $U_{\text {mean }}$ & Mean Velocity $\left(\mathrm{m}^{-1}\right)$ \\
$\mathrm{F}_{\mathrm{L}}$ & Lift force $\left(\mathrm{kgms}^{-2}\right)$ & $\mathrm{z}$ & Axial direction \\
$\mathrm{p}$ & Fluid pressure $\left(\mathrm{kgm}^{-1} \mathrm{~s}^{-2}\right)$ & $\rho$ & Density of fluid \\
$\mathrm{r}$ & Radical direction $(\mathrm{m})$ & $\mu$ & $\begin{array}{l}\text { Reference viscosity of fluid }(\mathrm{kg} \\
\left.\mathrm{m}^{-1} \mathrm{~s}^{-1}\right)\end{array}$ \\
$\mathrm{t}$ & Time(sec) & $\vartheta$ & Kinematic viscosity $\left(\mathrm{m}^{2} \mathrm{~s}^{-1}\right)$ \\
$\mathrm{u}$ & $\begin{array}{l}\text { The velocity of the fluid } \\
\left.\text { (ms }{ }^{-1}\right)\end{array}$ & $\nabla$ & Gradient operator $\left(\mathrm{m}^{-1}\right)$ \\
$u_{r}$ & Radial velocity $\left(\mathrm{ms}^{-1}\right)$ & $\theta$ & Tangential direction $(\mathrm{rad})$ \\
$u_{\theta}$ & Tangential velocity $\left(\mathrm{ms}^{-1}\right)$ & $\mathrm{k}$ & Thermal Conductivity $(\mathrm{W} / \mathrm{m} . \mathrm{K})$ \\
$C_{p}$ & Heat Capacity $\left(\mathrm{J} / \mathrm{kg}^{-\mathrm{K}}\right)$ & $\gamma$ & Heat Capacity ratio \\
\hline
\end{tabular}




\section{Introduction:}

A smooth straightforward flow, which does not show any zigzagging is called laminar flow. Small Reynolds number possesses laminar flow. Higher Reynolds numbers, cause a transition to be turbulence. Usually, the critical Reynolds number is known to be around 200.

GB Schubauer and HK Skramstad [1] confirmed the theory, putting an end to a long-running debate and shedding new light on the causes of the transition from laminar to turbulent flow. The Naiver-Stokes equations were solved by Abraham S. Berman [2] for a complete description of fluid flow in a channel with a rectangular cross-section and two equally porous walls. A practical aerodynamically and structurally reasonable efficient laminar flow control (LFC) suction method is developed and then applied to a second F94 LFC wing glove in-flight, removing the slowest boundary layer particles through many closed spaced fine slots: Up to the F94 test limit, 100 percent laminar flow is observed by Pfeminger, W. [3]. The history of aircraft laminar flow control (LFC) from the 1930s to the 1990s, as well as the current state of the technology, is examined by Joslin, R. D. [4]. Schrauf, G. [5] studied natural laminar flow (NLF) and hybrid laminar flow control (HLFC). An incompressible Naiver-Stokes equation developed within the Priority Research Program is provided by M. Schafer et al [6] for 2D and 3D laminar flow.

Fluids whose density varies significantly when applied pressure changes are dealt with by the branch of mechanics known as Compressible flow. If flow's Mach number exceeds 0.3 then flow is considered significantly compressible.

The principles of classical compressible flow have been used to solve problems in fields as diverse as high-speed aerodynamics and long-distance transport of low-speed gases by M. A Saad [7]. TaiPing Liu et al [8] Investigate the evolutions of gas-vacuum interfaces for both in-viscid and viscous one-dimensional isentropic gas motions. For Upgrading the worth of time-dependent solutions of the Euler equations in two dimensions Peraire et al. [9] described an adaptive mesh technique. Thomas H. Pulliam and Joseph L. Steger [10] adaptive an implicit finite-difference procedure for unsteady three-dimensional flow. Artificial boundary conditions (BCs) for simulation of inflow, outflow, and far-field (radiation) problems, with a focus on compressible turbulent shear flow techniques, are reviewed by Colonius, T. [11]. Subgrid models for Large Eddy Simulation (LES) of compressible turbulent flow are tested by Bert Verman et al. [12] for the three-dimensional mixing layer.

A flow is said to be time-dependent if field variables change over time. The time-dependent study is used in electromagnetism, heat transfer, and solid mechanics.

Kiseok Sunga et al. [13] presented a new scheme for unsteady networks as well as a solution algorithm based on Dijkstra's label setting algorithm. A new technique for numerically investigating the Unsteady flow of an incompressible fluid with an invidiously confined and invidiously free boundary is exposed by Francis H. Harlow and J. Eddie Welch [14]. To generate particle traces in unsteady flow fields, a particle tracing system has been developed by D.A Lane [15]. The dispersion of a non-uniform slug in time-variable fully constructed laminar flow is 
authorized by Gill, W.N. [16]. T. Lolla et al. [17] create and demonstrate the methodology for predicting the time-optimal paths of ocean vehicles in continuous dynamic flows. Volker John [18] describes a numerical investigation of a two-dimensional time-dependent flow around a cylinder. The Rayleigh number at which steady convective flow transitions to time-dependent flow is experimentally determined for several fluids with Prandtl numbers ranging from 1 to $10^{4}$ by Ruby Krishnamurti [19]. D. A Lane [20] present a method for visualizing time-dependent flow fields with streak lines. Kuangyu Shi [21] describe a method for visually analyzing the dynamic behavior of 3D time-dependent flow fields using path line behavior.

The current study reveals the numerical simulation of two-dimensional unsteady compressible laminar flow passing through a squared cylinder. Velocity and pressure distributions plots are presented for two-dimensional laminar steam flow. The results of the drag coefficient, heat distribution, lift coefficients are also investigated. The flow has a Reynolds number of approximately150, which is sufficient to keep the flow laminar.

\section{Mathematical formulation}

The region of flow is demonstrated in figure 1. First, the squared rectangular cylindrical geometry is constructed in COMSOL, whose length is $1 \mathrm{~m}$, breadth is $4 \mathrm{~m}$, then a square of length $0.15 \mathrm{~m}$ has been drawn at position $(.25 \mathrm{~m}, .2 \mathrm{~m})$. Then the difference between these two figures is made and then constructed all region is highlighted. In the next stage, in the section of material properties, the properties of the fluid are added. In Fig. 1, the boundary layers possess velocity $u$ zero. The boundary condition is considered no-slip and the initial pressure kept is zero. In the inlet, a velocity of $1 \mathrm{~m} / \mathrm{s}$ is given to flow to proceed and outlet condition is selected in COMSOL. For solving Naiver-Stokes equations, the physics of flow is added in COMSOL. Fig.1 shows the boundaries of the region of flow.

The position of rectangle and squared cylinder are as follows:

Rectangle: Position $(0,0)$ Width $(4 \mathrm{~m})$; Height $(1 \mathrm{~m})$ and size $(4,1)$

square: Position $(.25,0.2)$ sides $(0.15)$

The boundary conditions on the outer boundary are as follow:

Region ‘domain'Start ‘outer' $(0,0)$

\{mirror condition on bottom boundary

Natural $\left(u_{r}\right)=0$, Natural $\left(u_{\theta}\right)=0$, value $\mathrm{u}_{\mathrm{z}}=0$. 


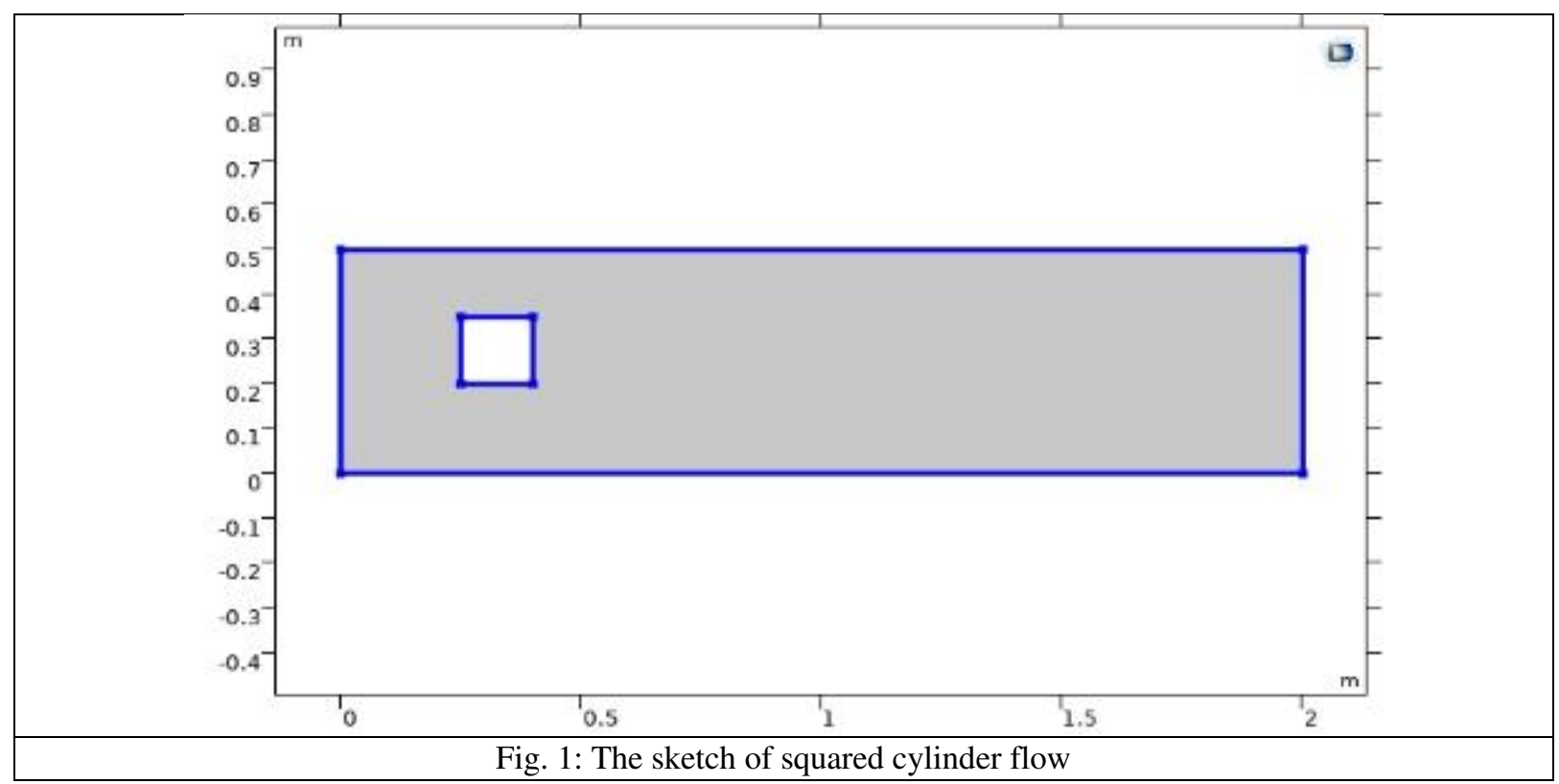

Natural $(\mathrm{p})=0$, line close to point $(2,0)$

No slip on sides (i.e., velocity $=0$ ) Value $u_{r}=0$, value $u_{\theta}=0, u_{z}=0$.

Natural $(p)=0$ line close to point $(2,0.5)$ Natural $\left(\mathrm{u}_{\mathrm{r}}\right)=0$, natural $\left(u_{\theta}=0\right)$,

Value $\left(u_{r}\right)=0$, natural $(p)=0$. line to close $(0,0.5)$

At $\mathrm{t}=0 ; \mathrm{u}_{\mathrm{r}}=0, u_{\theta}=0, \mathrm{u}_{\mathrm{z}}=0, \mathrm{P}=0$.

At $r=0.2, z=0.25, u=-U_{o} n$.

The drag and lift coefficients are defined as:

$C_{D}=\frac{2 F_{D}}{\rho U_{\operatorname{men}}^{2} D}, \quad C_{L}=\frac{2 F_{L}}{\rho U_{\operatorname{men}}^{2} D}$

The equation of continuity is:

$$
\frac{\partial p}{\partial t}+\nabla \cdot(\rho u)=0,
$$

The equation of momentum and heat equation is: 


$$
\rho C_{p}\left(\frac{\partial T}{\partial t}+u \cdot \nabla T\right)+\nabla \cdot\left(q+q_{r}\right)=\alpha_{p} T\left(\frac{\partial P}{\partial t}+u \cdot \nabla P\right)+\tau \cdot \nabla u+Q
$$

$$
\begin{aligned}
& \rho \frac{\partial u}{\partial t}+\rho(u \cdot \nabla) u=\nabla \cdot[-p i+k]+F, \\
& \text { where } \mathrm{K}=\mathrm{u}\left[\nabla u+(\nabla u)^{T}\right]-\frac{2}{3} u(\nabla \cdot u) u .
\end{aligned}
$$

The Continuity and momentum equations will reduce to the following forms:

$$
\begin{aligned}
& \frac{\partial U_{r}}{\partial r}+\frac{1}{r} U_{r}+\frac{\partial U_{z}}{\partial z}=0, \\
& \frac{\partial u_{r}}{\partial t}+u_{r} \frac{\partial u_{r}}{\partial r}+u_{z} \frac{\partial u_{r}}{\partial z}-\frac{u_{\theta}^{2}}{r}=-\frac{1}{\rho} \frac{\partial P}{\partial r}+\vartheta \frac{\partial^{2} u_{r}}{\partial r^{2}}+\vartheta\left(\frac{\partial^{2} u_{\theta}}{\partial r \partial z}+\frac{\partial^{2} u_{r}}{\partial z^{2}}\right), \\
& \frac{\partial u_{\theta}}{\partial t}+u_{r} \frac{\partial u_{\theta}}{\partial r}+u_{z} \frac{\partial u_{\theta}}{\partial z}+u_{r} u_{\theta}=\vartheta\left[\frac{\partial^{2} u_{r}}{\partial z^{2}}+\frac{\partial^{2} u_{z}}{\partial r \partial z}\right]+\vartheta \frac{\partial^{2} u_{z}}{\partial z^{2}}-\frac{1}{\rho} \frac{\partial P}{\partial z}, \\
& \frac{\partial u_{z}}{\partial t}+u_{r} \frac{\partial u_{z}}{\partial r}+u_{z} \frac{\partial u_{z}}{\partial z}=\vartheta\left[\frac{\partial^{2} u_{r}}{\partial z^{2}}+\frac{\partial^{2} u_{z}}{\partial r \partial z}\right]+\vartheta \frac{\partial^{2} u_{z}}{\partial z^{2}}-\frac{1}{P} \frac{\partial P}{\partial z} .
\end{aligned}
$$

The transformations used for heat equation are

$$
\alpha_{p}=-\frac{1}{\rho} \frac{\partial P}{\partial T}, \sigma=-\rho I+\tau, Q_{p}=\alpha_{p} T\left[\frac{\partial P}{\partial t}+u . \nabla P\right], Q_{v d}=\tau . \Delta u, q=-d_{m} k \Delta T, d_{m}=1 m^{2}
$$

After transformation we get,

$$
\begin{array}{|l|}
\operatorname{Pr}\left(\theta^{\prime \prime}\right)=v\left(\frac{1}{2} \theta^{\prime} \eta+2 \theta s\right)-p r \frac{G v}{\mathrm{k}_{\mathrm{f}}\left(\mathrm{T}_{\mathrm{w}}-\mathrm{T}_{\infty}\right)}\left[\left\{\frac{\mathrm{q}_{0}}{\mathrm{~A}_{\mathrm{s}} \Delta \mathrm{T}}\right\}-\mathrm{p}\left\{\frac{\mathrm{u}_{\mathrm{r}}}{\mathrm{r}}+\frac{\partial \mathrm{u}_{\mathrm{r}}}{\partial \mathrm{r}}+\frac{\partial \mathrm{u}_{\theta}}{\partial \mathrm{z}}\right\}-\frac{1}{3}\left\{\frac{\partial \mathrm{u}_{\mathrm{z}}}{\partial \mathrm{z}}\right.\right. \\
+ \\
+\operatorname{pr} \frac{1}{\mathrm{k}_{\mathrm{f}}\left(\mathrm{T}_{\mathrm{w}}-\mathrm{T}_{\infty}\right)} \frac{1}{\mathrm{R}_{\mathrm{e}}}\left[\frac{\partial^{3}}{\partial \mathrm{r}^{3}}\left(\frac{4}{3} \mathrm{u}_{\mathrm{r}}+\mathrm{u}_{\theta}+\mathrm{u}_{\mathrm{z}}\right)+\frac{1}{3}\left(\frac{\mathrm{u}_{\theta}}{\mathrm{r}} \frac{\partial^{2} \mathrm{u}_{\theta}}{\partial^{2} \mathrm{r}}+\frac{\partial \mathrm{u}_{\mathrm{r}}}{\partial \mathrm{z}} \frac{\partial^{2} \mathrm{u}}{\partial^{2} \mathrm{r}}\right.\right. \\
\left.+\frac{4}{3}\left(\frac{\mathrm{u}_{\mathrm{r}}}{\mathrm{r}}\right)+\frac{4}{3} \frac{\partial \mathrm{u}_{\theta}}{\partial \mathrm{z}}\right]
\end{array}
$$




\section{The procedure of numerical solution}

In the solution, a finer mesh controlled by physics is used. The total number of triangular elements is 25314 . The number of quadrilateral elements is 1812 , the number of edge elements is 990 , and the number of vertex elements is 8 . The maximum size of the element is 0.014 , while the minimum element size is 0.0004 .

In COMSOL, the corner refinement option is selected for domain 1. Trimming options are available for dealing with sharp corners. The laminar flow section is used to investigate this model. Table 1 describes mesh statistics. Table 2, on the other hand, describes the mesh size. The numerical solution employs a normal mesh.

Table 1: The thermophysical properties of steam flow are as follow:

\begin{tabular}{lll}
\hline Property & Expression & Description \\
\hline Density & $0.590 \mathrm{~kg} / \mathrm{m}^{3}$ & Density of fluid \\
Viscosity & 0.68 pas. Second & The viscosity of the fluid \\
Temperature & $32{ }^{\circ} \mathrm{C}$ & Temperature during flow \\
\hline
\end{tabular}

Table 2: Description of mesh statistics and mesh size:

\begin{tabular}{|c|c|c|c|}
\hline Statistics & Value & Mesh & Size \\
\hline $\begin{array}{l}\text { Minimum quality of } \\
\text { the element }\end{array}$ & 0.4319 & Geometric Level of entry & Boundary \\
\hline $\begin{array}{l}\text { Average quality of } \\
\text { element }\end{array}$ & 0.8433 & Selection & $\begin{array}{l}\text { Boundaries 2-3,5- } \\
8\end{array}$ \\
\hline Triangular elements & 4038 & Calibrate for & Fluid dynamics \\
\hline $\begin{array}{l}\text { Quadrilateral quality } \\
\text { of element }\end{array}$ & 0.8358 & Maximum size of element & 0.067 \\
\hline Edge elements & 330 & $\begin{array}{l}\text { Minimum size of the } \\
\text { element }\end{array}$ & 0.003 \\
\hline Vertex & 8 & Factor of curvature & 0.4 \\
\hline Predefined size & Normal & $\begin{array}{l}\text { Maximum growth of } \\
\text { element rate }\end{array}$ & 1.2 \\
\hline
\end{tabular}




\section{Discussion and outcomes}

The results presented here demonstrate the laminar flow of steam in squared cylindrical geometry. The flow model has 2D geometry. The diameter of cylindrical flow is on the vertical axis, and the z-direction in the current two-dimensional plots is concerned by the horizontal axis. Fig. 2 represents velocity distribution at time $=2 \mathrm{~s}$. This figure represents the movement of fluid that strikes to a squared figured block kept in the path of flow. This figure shows that velocity is zero at boundary layers. The upper layers of the bottom boundary of the cylinder show peak values of velocity which is $1 \mathrm{~m} / \mathrm{s}$ until fluid strikes the squared figure block kept in the flow of fluid. At the point of contact of fluid and squared figure block velocity becomes again zero. The layers separating from the squared figure block again show enhanced values of velocities up to the top boundary of the cylinder.

Fig. 3 shows velocity distribution at time $=5 \mathrm{~s}$. This figure describes approximately the same behavior as does Fig. 2 except the fluid behavior which is moving away from the squared figure block. This part represents an enhanced change in velocity rather than the fluid flowing at time $=2$ s. The remaining layers possess velocities between $0 \mathrm{mls}$ and $1 \mathrm{mls}$. Fig. 4 and 5 represent fluid flow in the extents of flow at time $7 \mathrm{~s}$ and $10 \mathrm{~s}$ respectively. In Fig. 4 boundary layers show zero velocity. As we move from bottom to upward, the layers show an increase in velocity. At the center, velocity again becomes zero. And again an increasing velocity can be seen from the upper layers of central flow which ultimately becomes zero at the top of cylindrical geometry. Fig. 5 shows approximately the same oscillations of velocity as shown in Fig. 4. The difference is that in Fig. 4 velocity changes occur more rapidly than Fig. 5.

Fig. 6 and Fig. 7 show pressure distribution around squared figure block at time intervals $2 \mathrm{~s}$ and $5 \mathrm{~s}$ respectively. At the initial point, pressure shows maximum values by each layer. Whereas, at the connecting point of fluid and block different values of pressure can be seen. When the fluid strikes to block then upper and lower layers of fluid lose down their pressure and their pressure becomes zero. The layers moving apart from the block exhibit less decrease in their pressure. In Fig. 6 when fluid flows away from the block, pressure again goes on increasing. In Fig. 7 the layers moving away from the block but nearer to it maintain their pressure, but the layers which have some distance from the block show a slight increase in their pressure. The maximum values of pressure in Fig. 6 and Fig. 7 are 0.00098 Pas and 0.0010 Pas, respectively. The minimum value of pressure in Fig. 6 is -0.000134 and it is -0.000121 in Fig. 7.

Fig. 8 and Fig. 9 reveals pressure distribution in the outer part of flow at time intervals $7 \mathrm{~s}$ and 10 s respectively. In Fig. 8 at starting point fluid shows zero pressure at the center. when flow proceeds, an increase in pressure can be seen. Fig. 9 follows the same pattern, but an increase in pressure is achieved at the outer boundary. The maximum value of pressure in figure 8 is 0.00099 Pas and in figure 9 maximum value of pressure is 0.000101 Pas. The minimum value of pressure in Fig. 8 is -0.000147 and it is -0.000155 Pas in Fig. 9. Fig. 10 and 11 show surface temperature around the squared figure block kept in the flow of fluid at time intervals $t=2 \mathrm{~s}$ and $\mathrm{t}=5 \mathrm{~s}$, 
respectively. Boundary layers of fluid show the maximum value of temperature throughout the flow which is $360 \mathrm{~K}$ in Fig. 10 and $370 \mathrm{~K}$ in Fig. 11. The middle layers of flow show minimum values of temperature which is $232 \mathrm{~K}$ in Fig. 10 and $280 \mathrm{~K}$ in Fig. 11. The other remaining layers oscillate between these maximum and minimum values.

Fig. 12 and Fig. 13 describe surface temperature in the outer part of flow at time intervals $7 \mathrm{~s}$ and 10 s respectively. Fig 12 and Fig. 13 show approximately the same temperature behavior of Fig. 10 and Fig. 11. The difference between these two figures is the maximum and minimum values of temperature. The maximum temperature of Fig. 12 and Fig. 13 is $375 \mathrm{~K}$. Minimum temperature in Fig. 12 is $306 \mathrm{~K}$ and in Fig. 13 it is $334 \mathrm{~K}$. Fig. 14 and Fig. 15 show contour temperature around squared block at time intervals $2 \mathrm{~s}$ and $5 \mathrm{~s}$. These figures show that flow possesses minimum values of contour temperature by boundary layers. Minimum contour temperatures are $235.3 \mathrm{~K}$ and 282.7 $\mathrm{K}$ in Fig. 14 and Fig. 15, respectively. Maximum values of contour temperature in Fig. 14 and Fig. 15 are $371.56 \mathrm{~K}$ and $372.7 \mathrm{~K}$.

Fig. 16 and 17 describe the contour temperature in the outer edge of the squared cylinder at time $\mathrm{t}=7 \mathrm{~s}$ and $\mathrm{t}=10 \mathrm{~s}$, respectively. These figures show that boundary layers possess maximum values of contour temperature while middle layers show minimum values of contour temperature. The remaining layers show oscillation of contour temperature between maximum and minimum values. Maximum values for contour temperature are $373.42 \mathrm{~K}$ and $374.12 \mathrm{~K}$ in figure 16 and Fig. 17 respectively. The minimum value of contour temperature in Fig. 16 is $307.72 \mathrm{~K}$ and it is $334.45 \mathrm{~K}$ in Fig. 17. Fig. 18 shows a table graph of drag coefficient in cylindrical flow. The graph is plotted between time and drag coefficient. This figure shows that with an increase in time, an increase in drag coefficient is also achieved. In a time interval of $10 \mathrm{~s}$ drag coefficient increases from 0 to 0.065 .

Fig. 19 represents a surface graph of drag coefficient in cylindrical flow. This figure shows that when fluid comes in contact with a squared block then the maximum value of drag coefficient is produced which ultimately becomes zero. The maximum value of the drag coefficient in this figure is 10 .

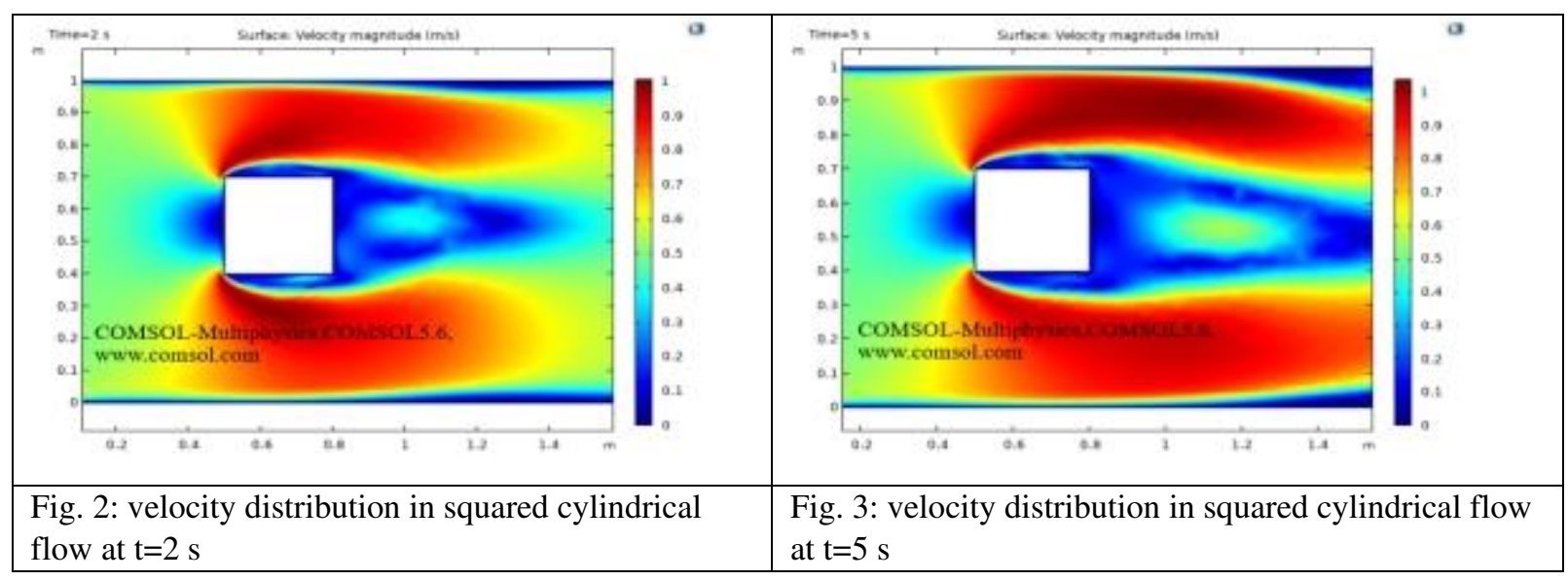




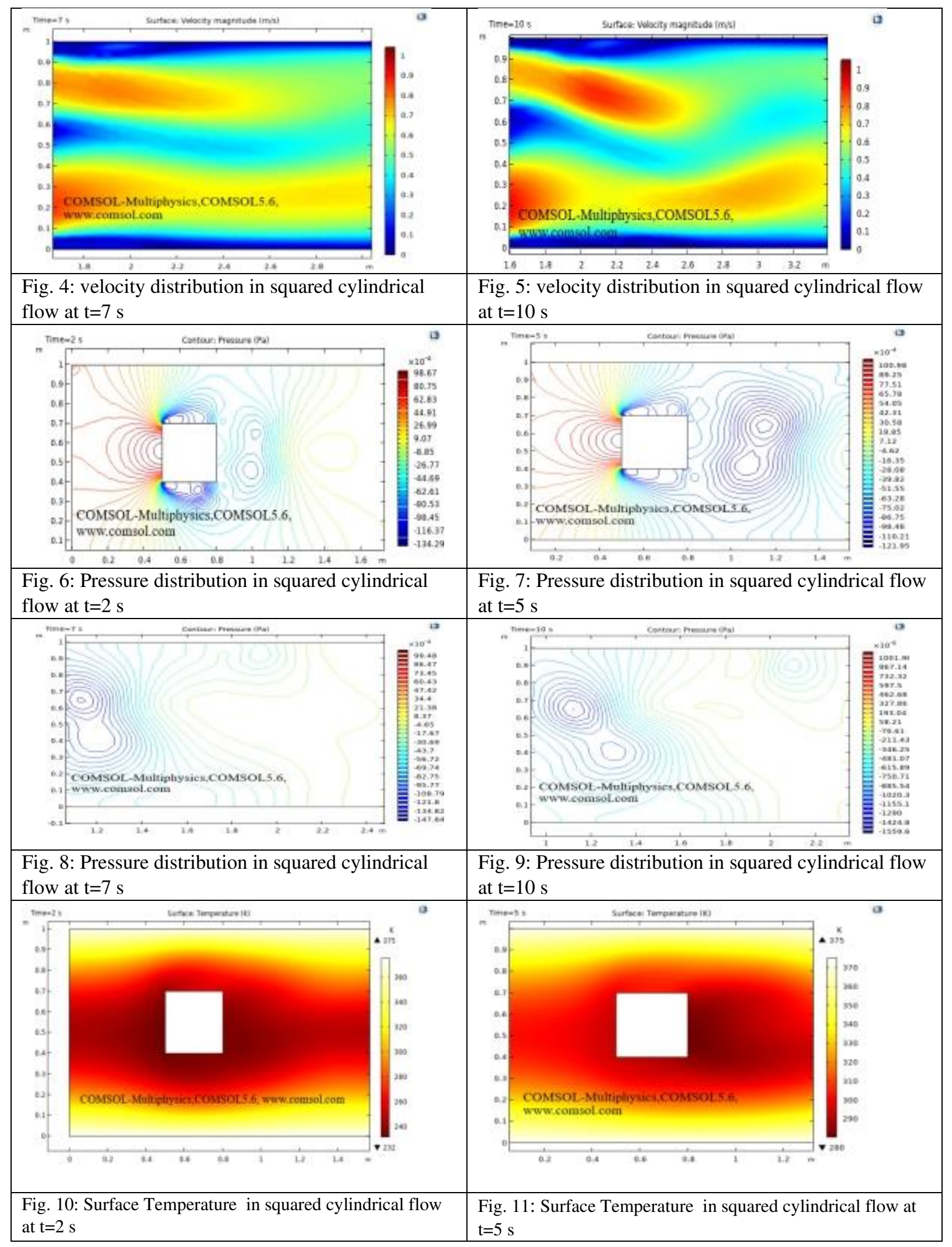




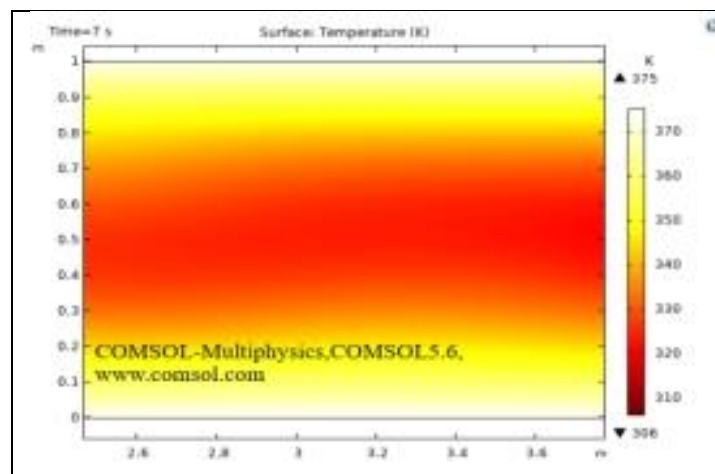

Fig. 12: Surface Temperature in squared cylindrical flow at $\mathrm{t}=7 \mathrm{~s}$

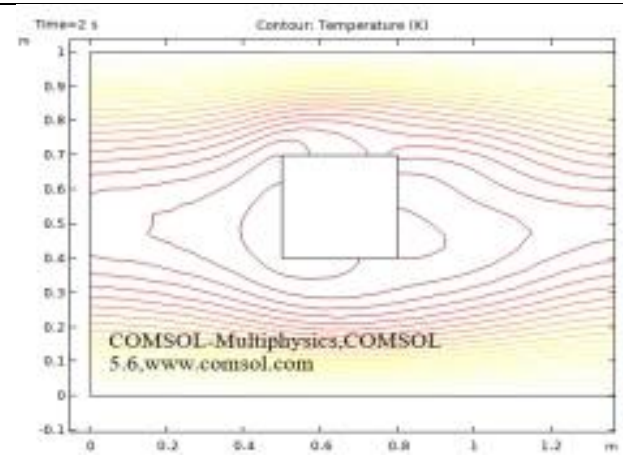

Fig. 14: Contour temperature in squared cylindrical flow at $\mathrm{t}=2 \mathrm{~s}$
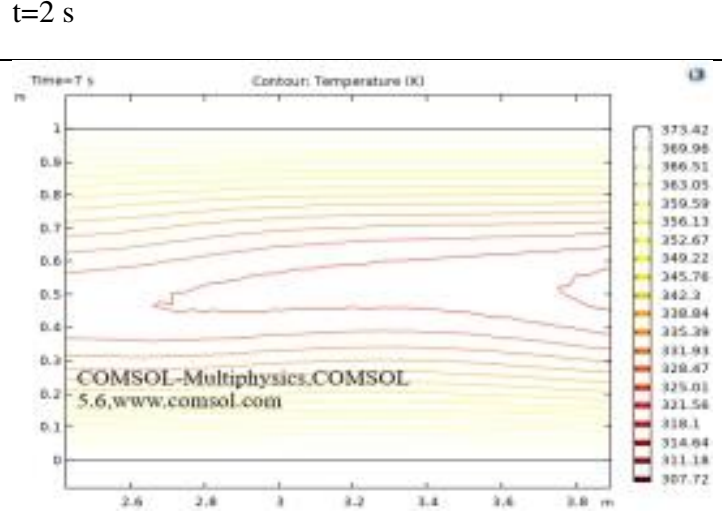

Fig. 16: Contour temperature in squared cylindrical flow at $\mathrm{t}=7 \mathrm{~s}$

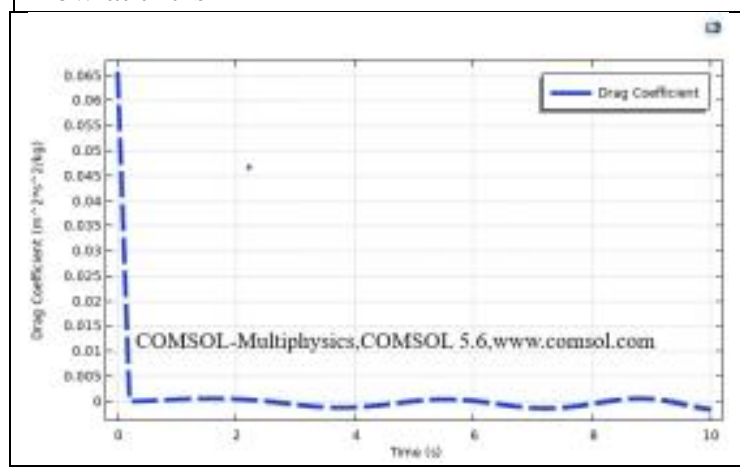

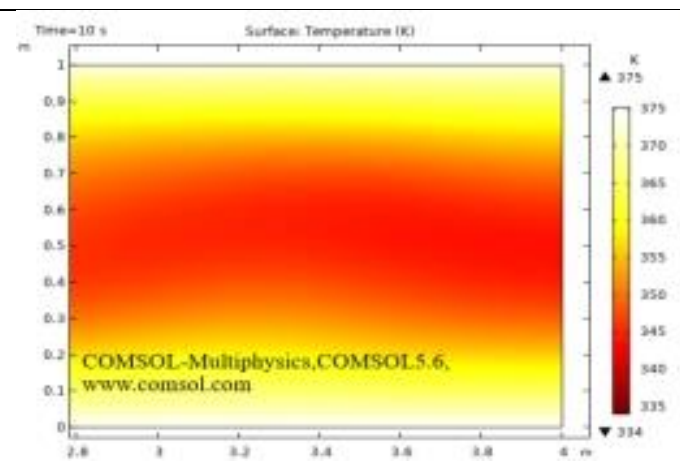

Fig. 13: Surface Temperature in squared cylindrical flow at $\mathrm{t}=10 \mathrm{~s}$

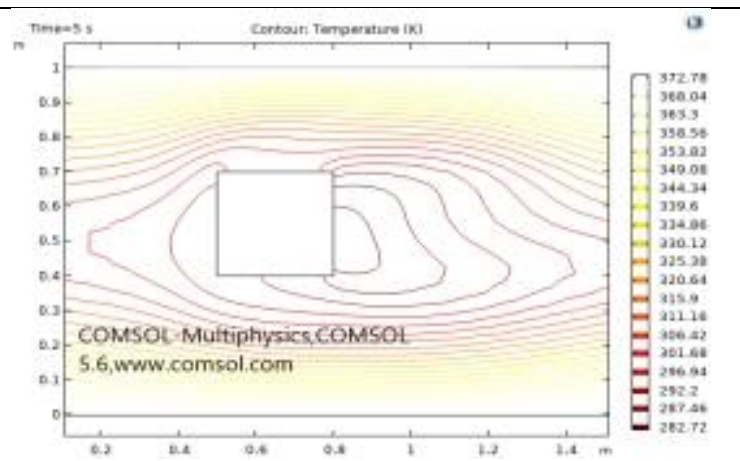

Fig. 15: Contour temperature in squared cylindrical flow at $\mathrm{t}=5 \mathrm{~s}$

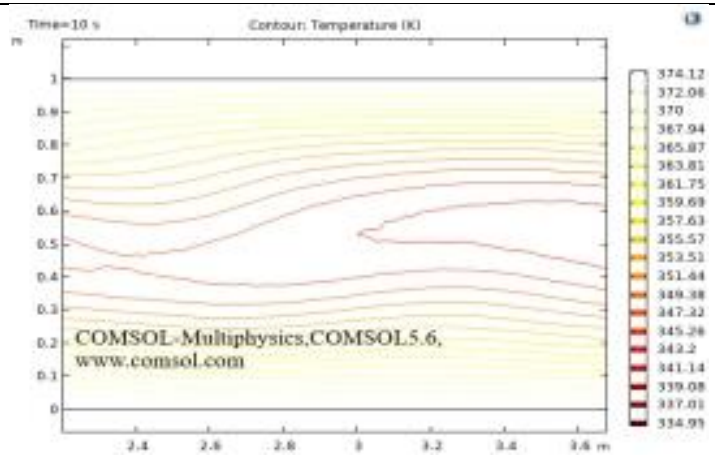

Fig. 17: Contour temperature in squared cylindrical flow at $\mathrm{t}=10 \mathrm{~s}$

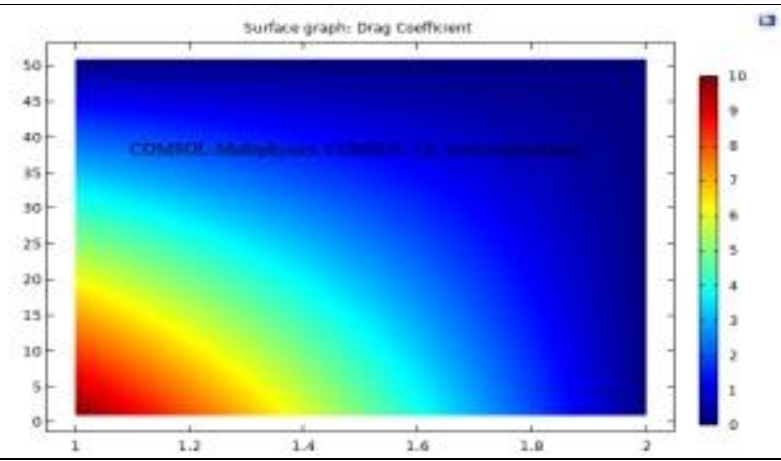




\section{Conclusion}

The current study highlights the calculated simulation of unsteady two-dimensional fluid flow passing through a squared cylinder. Plots of velocity and pressure distributions are served. Results of heat distribution are also given graphically. The results of the drag and lift coefficients are authorized as well. The flow has a Reynolds number of approximately 150, which is sufficient to maintain the laminar flow. A higher Reynolds number in such a flow causes the laminar flow to be turbulent. A high Reynolds number reduces the drag coefficient as well. When the Mach number is less than 0.3 , the flow is compressible. The following are the main findings of the current study:

1. The velocity drops from $2.5 \mathrm{~m} / \mathrm{s}$ to $1.6 \mathrm{~m} / \mathrm{s}$ at $\mathrm{t}=7 \mathrm{~s}$ in the absence of anybody's force and temperature $305.13 \mathrm{~K}$.

2. Pressure increases from 0.00098 Pas to 0.001 Pas in the flow interval of $10 \mathrm{~s}$.

3. Surface temperature increases from $360 \mathrm{~K}$ to $375 \mathrm{~K}$ in time intervals of 10 s keeping pressure constant.

4. Contour temperature increases from $371.56 \mathrm{~K}$ to $374.2 \mathrm{~K}$ in time intervals of 10 s keeping pressure constant.

5. The lift coefficient remains constant and the drag coefficient increases from 0.0005 to 0.065 .

\section{References}

[1].Schubauer, G. B., \& Skramstad, H. K. (1947). Laminar boundary-layer oscillations and stability of the laminar flow. Journal of the Aeronautical Sciences, 14(2), 69-78.

[2]. Pfeminger, W. (1977). Laminar flow control laminarization.

[3]. Joslin, R. D. (1998). Aircraft laminar flow control. Annual review of fluid mechanics, 30(1), 129.Issa, R. I., Gosman, A. D., \& Watkins, A. P. (1986). The computation of compressible and incompressible recirculating flows by a non-iterative implicit scheme. Journal of Computational Physics, 62(1), 66-82.

[4]. Schrauf, G. (2005). Status and perspectives of laminar flow. The aeronautical journal, 109(1102), 639-644.

[5]. Schäfer, M., Turek, S., Durst, F., Krause, E., \& Rannacher, R. (1996). Benchmark computations of laminar flow around a cylinder. In Flow simulation with high-performance computers II (pp. 547-566). Vieweg+ Teubner Verlag.

[6]. Saad, M. A. (1985). Compressible fluid flow. Englewood Cliffs..

[7]. Liu, T. P., Xin, Z., \& Yang, T. (1998). Vacuum states for compressible flow. Discrete \& Continuous Dynamical Systems, 4(1), 1.

[8]. Peraire, J., Vahdati, M., Morgan, K., \& Zienkiewicz, O. C. (1987). Adaptive remeshing for compressible flow computations. Journal of computational physics, 72(2), 449-466.. 
[9]. Pulliam, T. H., \& Steger, J. L. (1980). Implicit finite-difference simulations of threedimensional compressible flow. AIAA Journal, 18(2), 159-167.

[10]. Colonius, T. (2004). Modeling artificial boundary conditions for compressible flow. Annu. Rev. Fluid Mech., 36, 315-345.

[11]. Vreman, B., Geurts, B., \& Kuerten, H. (1995). Subgrid-modelling in LES of compressible flow. Applied scientific research, 54(3), 191-203.

[12]. Sung, K., Bell, M. G., Seong, M., \& Park, S. (2000). Shortest paths in a network with timedependent flow speeds. European Journal of Operational Research, 121(1), 32-39.

[13]. Harlow, F. H., \& Welch, J. E. (1965). Numerical calculation of time-dependent viscous incompressible flow of fluid with free surface. The physics of fluids, 8(12), 2182-2189.

[14]. Lane, D. A. (1994, October). UFAT-a particle tracer for time-dependent flow fields. In Proceedings Visualization'94 (pp. 257-264). IEEE.

[15]. Gill, W. N., Sankarasubramanian, R., \& Taylor, G. I. (1971). Dispersion of a non-uniform slug in time-dependent flow. Proceedings of the Royal Society of London. A. Mathematical and Physical Sciences, 322(1548), 101-117.

[16]. Lolla, T., Ueckermann, M. P., Yiğit, K., Haley, P. J., \& Lermusiaux, P. F. (2012, May). Path planning in time dependent flow fields using level set methods. In 2012 IEEE International Conference on Robotics and Automation (pp. 166-173). IEEE.

[17]. John, V. (2004). Reference values for drag and lift of a two-dimensional time-dependent flow around a cylinder. International Journal for Numerical Methods in Fluids, 44(7), 777-788.

[18]. Krishnamurti, R. (1970). On the transition to turbulent convection. Part 2. The transition to time-dependent flow. Journal of Fluid Mechanics, 42(2), 309-320.

[19]. Lane, D. A. (1993, October). Visualization of time-dependent flow fields. In Proceedings Visualization'93 (pp. 32-38). IEEE.

[20]. Shi, K., Theisel, H., Hauser, H., Weinkauf, T., Matkovic, K., Hege, H. C., \& Seidel, H. P. (2009).

[21] Path line attributes-an information visualization approach to analyzing the dynamic behavior of 3D time-dependent flow fields. In Topology-Based Methods in Visualization II (pp. 7588). Springer, Berlin, Heidelberg. 